\title{
Efficacy and Safety of Daclatasvir and Asunaprevir in Patients with Hepatitis C Virus Genotype 1b Infection on Hemodialysis
}

\author{
Byung Seok Lee ${ }^{1}$, Myeong Jun Song ${ }^{2}$, Jung Hyun Kwon ${ }^{3}$, Tae Hee Lee ${ }^{4}$, Ji Woong Jang ${ }^{5}$, Seok Hyun Kim ${ }^{1}$, Sae Hwan Lee $^{6}$, \\ Hong Soo Kim ${ }^{6}$, Ji Hoon $\mathrm{Kim}^{7}$, Seok Bae Kim ${ }^{8}$, Soon Young Ko ${ }^{9}$, and Do Seon Song ${ }^{10}$ \\ ${ }^{\prime}$ Department of Internal Medicine, Chungnam University College of Medicine, ${ }^{2}$ Division of Hepatology, Department of Internal Medicine, \\ Daejeon St. Mary's Hospital, College of Medicine, The Catholic University of Korea, Seoul, ${ }^{3}$ Division of Hepatology, Department of Internal \\ Medicine, Incheon St. Mary's Hospital, College of Medicine, The Catholic University of Korea, Seoul, ${ }^{4}$ Department of Internal Medicine, \\ Konyang University College of Medicine, ${ }^{5}$ Department of Internal Medicine, Eulji University Hospital, Eulji University College of Medicine, \\ Daejeon, ${ }^{6}$ Department of Internal Medicine, Soonchunhyang University Hospital, Soonchunhyang University College of Medicine, Cheonan, \\ ${ }^{7}$ Department of Internal Medicine, Korea University Guro Hospital, Korea University College of Medicine, Seoul, ${ }^{8}$ Department of Internal \\ Medicine, Dankook University Hospital, Dankook University College of Medicine, Cheonan, ${ }^{9}$ Department of Internal Medicine, Konkuk \\ University School of Medicine, Seoul, and ${ }^{10}$ Division of Hepatology, Department of Internal Medicine, St. Vincent's Hospital, College of \\ Medicine, The Catholic University of Korea, Seoul, Korea
}

Background/Aims: We evaluated the efficacy and safety of daclatasvir (DCV) and asunaprevir (ASV) in patients with chronic hepatitis $\mathrm{C}$ virus (HCV) infection on hemodialysis. Methods: We performed a single-arm, multicenter prospective study. Twenty-one chronic hemodialysis patients with $\mathrm{HCV}$ infection were prospectively enrolled from February 2016 to April 2017. We evaluated the virological responses at weeks 4, 12, and 24 (end of treatment [EOT]) and the sustained virological response at 12 weeks after the EOT (SVR12). The tolerability and safety of the drugs were also assessed. Results: None of the 20 patients had the NS5A resistance-associated variant (NS5A RAV), and one patient was indeterminate for the NS5A RAV. Seventeen patients (80\%) completed the 24 weeks of treatment with DCV and ASV. Four patients discontinued the study prior to week 12. In an intention-to-treat analysis, the SVR12 was $76.1 \%$. In a per-protocol analysis, patients who completed DCV and ASV treatment achieved an SVR12 of 100\%. DCV and ASV were well tolerated by the majority of patients. Three patients discontinued treatment due to adverse events (AEs) including dizziness, dyspnea, and neutropenia. The patient with indeterminate NS5A RAV showed viral breakthrough and discontinued treatment. Conclusions: DCV and ASV combination therapy in chronic hemodialysis patients with HCV infection achieved a high SVR12 rate with few AEs. To maximize the SVR12 rate, it is important to identify candidates by baseline
RAV testing. Close monitoring of the safety and tolerability of DCV and ASV may be necessary in HCV-infected patients on hemodialysis. (ClinicalTrials.gov ID NCT02580474) (Gut Liver 2019;13:191-196)

Key Words: Daclatasvir; Asunaprevir; Chronic hepatitis C virus; Hemodialysis; Sustained virologic response

\section{INTRODUCTION}

Hepatitis C virus (HCV) is a major cause of chronic hepatitis, liver cirrhosis, and hepatocellular carcinoma. ${ }^{1}$ The prevalence of HCV infection is approximately $2.8 \%$ and estimated 2.7 to 3.5 million people. ${ }^{2}$ Patients on hemodialysis, with human immunodeficiency virus infection, and intravenous drug abusers are at high risk of HCV infection. HCV prevalence of in the hemodialysis patients is reportedly $7.8 \%$ to $44 \%$ in the world. ${ }^{3-6}$

Chronic HCV infection is associated with an increased risk of end-stage renal disease (ESRD). ${ }^{7.8}$ A high HCV RNA level and HCV genotype 1 are predictive of ESRD. ${ }^{8}$ HCV-infected patients have a $23 \%$ greater risk of presenting with chronic kidney disease (CKD) compared to uninfected patients. ${ }^{7}$ In addition, CKD patients with HCV have the risk of progression of cirrhosis, hepatocellular carcinoma, and liver-related mortality compared to those without HCV infection. ${ }^{9}$ Therefore, active HCV treatment of patients with CKD infection is important.

The treatment of difficult-to-treat HCV-infected patients with

Correspondence to: Myeong Jun Song (https://orcid.org/0000-0001-5244-0372)

Division of Hepatology, Department of Internal Medicine, Daejeon St. Mary's Hospital, College of Medicine, The Catholic University of Korea, 64 Daeheung-ro, Jung-gu, Daejeon 34943, Korea

Tel: +82-42-220-9291, Fax: +82-42-252-6807, E-mail: mjsong95@gmail.com

Received on May 28, 2018. Revised on July 9, 2018. Accepted on August 13, 2018. Published online January 3, 2019

pISSN 1976-2283 eISSN 2005-1212 https://doi.org/10.5009/gnl18240

@) This is an Open Access article distributed under the terms of the Creative Commons Attribution Non-Commercial License (http://creativecommons.org/licenses/by-nc/4.0) which permits unrestricted non-commercial use, distribution, and reproduction in any medium, provided the original work is properly cited. 
ESRD is an important issue in clinical practice. These patients have more comorbidities and show a poor response to pegylated interferon-based treatment. ${ }^{10}$ Furthermore, the prognosis of hemodialysis patients is worsened by HCV infection. Although patients with ESRD have the chance to receive kidney transplantation, ESRD patients with HCV is limited to this opportunity and are at risk of progression of the underlying liver disease because of immune-suppressive therapy in post-transplantation state. ${ }^{11}$ Therefore, it is important to assess the efficacy and safety of new HCV therapeutics in subjects with renal impairment.

Direct antiviral agents (DAAs) are effective and safe in HCVinfected patients. A 24-week regimen of daclatasvir (DCV) and asunaprevir (ASV) was the first approval of DAAs in Korea 2015. This regimen yielded a post-end of treatment (EOT) sustained virological response at week 12 (SVR12) rate of up to 90\% in Korean population; this represented an important milestone in the treatment of HCV infection. ${ }^{12}$ In addition, pharmacokinetics of these DAAs showed that they are metabolized in the liver and excreted into the bile ducts. ${ }^{13,14}$ The elimination of DAAs is thus not affected by renal dysfunction. The combination therapy is available in HCV patient with hemodialysis.

However, there is lack of data of safety and efficacy of DAAs (especially DCV-ASV) available in patients with severe renal impairment (creatinine clearance $<30 \mathrm{~mL} / \mathrm{min}$ ) in recent $\mathrm{HCV}$ treatment guidelines. ${ }^{15-17}$ Therefore, we evaluated the efficacy and safety of DCV and ASV in patients with HCV infection on hemodialysis.

\section{MATERIALS AND METHODS}

\section{Study design and populations}

This open-label, multicenter, interventional, prospective single-arm study compared the efficacy and safety of the DCVASV combination with those of pegylated interferon and ribavirin.

We prospectively enrolled $21 \mathrm{HCV}$-infected ESRD patients on hemodialysis at 10 medical centers from February 2016 to April 2017. Subjects who met all of the following inclusion criteria were enrolled: (1) adult male and non-pregnant, non-lactating female subjects, aged $\geq 18$ years; (2) documented evidence of chronic HCV infection (e.g., HCV RNA-positive and HCV genotype 1b); (3) no history, sign or symptom of decompensated liver disease; (4) hemodialysis; (5) no baseline mutation NS5A polymorphism including $\mathrm{L} 31 \mathrm{~F} / \mathrm{I} / \mathrm{M} / \mathrm{V}$ and $\mathrm{Y93H}$; (6) HCV treatment-naïve, defined as never having received HCV treatment with an approved or investigational drug (including vaccines); (7) or HCV treatment-experienced, defined as having received HCV treatment with a pegylated interferon-based drug regimen (with or without ribavirin and not including DAAs). The exclusion criteria were: (1) evidence of a medical condition contributing to chronic liver disease other than HCV or seropositivity for human immunodeficiency virus or hepatitis B virus; (2) diagnosed or suspected hepatocellular carcinoma or other malignancy; (3) a history of, evidence of current clinical hepatic decompensation (e.g., ascites, encephalopathy or variceal hemorrhage); (4) receipt of solid organ or bone marrow transplant; (5) significant renal, cardiovascular, pulmonary, or neurological disease, or uncontrolled diabetes or hypertension; (6) currently receiving immune suppressive therapy (e.g., corticosteroids), investigational agents, nephrotoxic agents, or agents that affect renal excretion; (7) known hypersensitivity to the study drugs or their metabolites; and (8) current alcohol or substance abuse.

The study consisted of a 24-week treatment period followed by a 12-week posttreatment. Treatment regimens were fixed doses of DCV (60 mg once daily) and ASV (100 mg twice daily). The patients were followed-up at weeks 4, 12, and 24 (EOT) and weeks 12 after EOT. Physical examinations, tolerability, and laboratory tests were performed at each visit. Patient records were de-identified and anonymously analyzed. This study was conducted in accordance with the Declaration of Helsinki, and ethical approval was obtained from the Ethics Review Board of the Catholic University of Korea (XC15MIMV0076D). The study was registered with U.S. ClinicalTrials.gov (Trial ID: NCT ID NCT02580474).

\section{Endpoints and definitions}

The primary endpoints were SVR12 and safety. An SVR12 was defined as an HCV RNA level below the lower limit of quantification (15 IU/mL). Safety was evaluated by investigating adverse events (AEs) (e.g., anemia, skin rash) and discontinuation of the study medications.

The secondary endpoints were: virologic response at weeks 4, 12, and 24 (EOT); normalized alanine aminotransferase (ALT) level; and treatment failure including viral breakthrough and relapse. Any patient who met either of the following criteria was considered to have on-treatment virologic failure and was required to discontinue treatment: an increase in the HCV RNA level $>10^{2} \mathrm{IU} / \mathrm{mL}$ after having an undetectable HCV RNA level; or a confirmed increase in the HCV RNA $>1 \log _{10}$ from the nadir during the treatment period.

\section{Statistical analysis}

The primary endpoint of this study was the SVR12 rate, which was compared to that of pegylated interferon. Previous Korean retrospective study showed SVR12 rate was 65\% in genotype $1 \mathrm{HCV}$ patients with ESRD. ${ }^{18,19}$ Because there was no reference of SVR12 rate for the treatment of DAA in ESRD patients at that time of study, we estimated expected SVR12 rate based on the result of the Japanese study. In that study, DCV and ASV combination therapy showed SVR12 rate of 96\% in relapsed patient group. ${ }^{20}$ Therefore, we calculated and designed 95\% based on this result because our study group is difficult to treat HCV. We calculated that a sample size of at least 14 patients would provide $80 \%$ power to differentiate SVR12 rates of 
$65 \%$ and $95 \%$ by a two-sided test at a significance level of 0.05 .

The primary endpoint was analyzed after all enrolled patients had completed the week 12 posttreatment visit. We analyzed treatment efficacy and safety in intention-to-treat (ITT) and perprotocol (PP) populations. In the ITT analysis, we evaluated the SVR12 rate in all patients who received at least one dose of the study medication. The PP analysis was performed after excluding patients who discontinued treatment for administrative reasons.

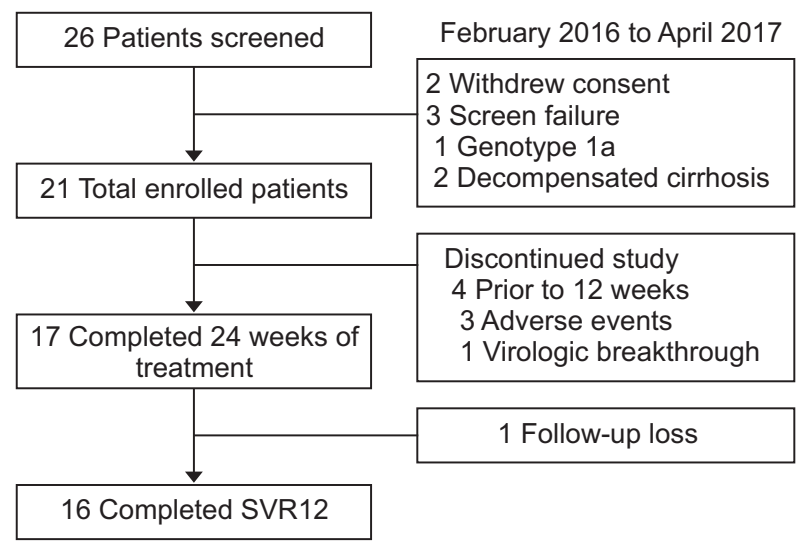

Fig. 1. Flowchart of patients enrolled in this study. SVR12, sustained virologic response 12 weeks posttreatment.

Table 1. Baseline Characteristics

\begin{tabular}{lc}
\hline \multicolumn{1}{c}{ Characteristics } & Value $(\mathrm{n}=21)$ \\
\hline HCV genotype & $1 \mathrm{~b}$ \\
Age, yr & $59(39-82)$ \\
Male sex & $13(61.9)$ \\
Comorbidities & \\
DM & $16(76.1)$ \\
HBP & $20(95.2)$ \\
Median HCV RNA, $\log _{10} \mathrm{IU} / \mathrm{mL}$ & $5.18(1.88-7.20)$ \\
Baseline Hb, g/dL & $11.5(9.3-14.3)$ \\
Baseline platelet, $\times 10^{3} / \mu \mathrm{L}$ & $138(55-274)$ \\
Baseline ALT, IU/L & $22(7-113)$ \\
HCV treatment history & \\
Naïve/experienced & $18(85.7) / 3(14.2)$ \\
Liver cirrhosis & $4(19.0)$ \\
Hemodialysis & $21(100)$ \\
NS5A RAVs mutation (no/indeterminate) & $20(95.2) / 1(4.8)$ \\
\hline
\end{tabular}

Data are presented as median (range) or number (\%).

DM, diabetes mellitus; HBP, high blood pressure; HCV, hepatitis C virus; $\mathrm{Hb}$, hemoglobin; ALT, alanine aminotransferase; RAVs, resistance-associated variants.

\section{RESULTS}

\section{Baseline characteristics of the study population}

Twenty-six HCV patients with hemodialysis were prospectively screened. Of them, 21 patients were enrolled in the study and other five were excluded due to screening failure $(n=3)$ and withdrawal of consent ( $n=2)$ (Fig. 1).

The baseline characteristics of the enrolled patients are shown in Table 1. All patients were infected with genotype $1 \mathrm{~b}$ HCV. The median age of the patients was 59 years, and 61.9\% $(n=13)$ were males. Four patients had compensated liver cirrhosis; the others had chronic hepatitis. In one patient, the presence of NS5A polymorphisms at baseline, including $\mathrm{L} 31 \mathrm{~F} / \mathrm{I} / \mathrm{M} / \mathrm{V}$ and $\mathrm{Y} 93 \mathrm{H}$, was indeterminate. The most frequent causes of renal dysfunction were diabetes mellitus (DM; 76.1\%, 16/21) and hypertension $(95.2 \%, 20 / 21)$. Three patients had previously received pegylated interferon-based therapy; the others were treatment-naïve.

\section{Treatment efficacy}

During follow-up, 17 patients with ESRD completed 24 weeks of combination therapy and 16 patients had a sustained virologic response at 12 weeks. One patient who had a virologic response (HCV RNA <15 IU/mL) at the EOT was lost to followup at 12 weeks posttreatment. In the ITT analysis, treatment with DCV and ASV for 24 weeks resulted in an SVR12 in 76.1\% of the patients (16/21 patients) (Table 2). Two patients were reported to have virologic breakthrough; the baseline NS5A mutation status of one was indeterminate and the other patient did not comply with the treatment due to AEs. In addition, two patients did not complete the treatment due to AEs not related to the study drug.

Five patients who discontinued the treatment for administrative reasons (baseline indeterminate NS5A mutation $[n=1]$; nonadherence due to AEs, considered unlikely to be related to the study drug by the investigator $[n=3]$; and loss to follow-

Table 2. Virologic Response by Intention-to-Treat Analysis ( $\mathrm{n}=21)$

\begin{tabular}{lc}
\hline \multicolumn{1}{c}{ Virologic response } & No. (\%) \\
\hline Week 4 & $20(95.2)$ \\
Week 12 & $18(85.7)$ \\
Week 24 (end of treatment) & $17(80.9)$ \\
SVR 12 & $16(76.1)$ \\
Virologic breakthrough & $2(9.5)$ \\
Viral relapse & 0 \\
SVR12 by NS5A RAV mutation & \\
No NS5A & $16(80)$ \\
Indeterminate NS5A & 0 \\
\hline
\end{tabular}

SVR12, sustained virologic response 12 weeks posttreatment; RAVs, resistance-associated variants. 


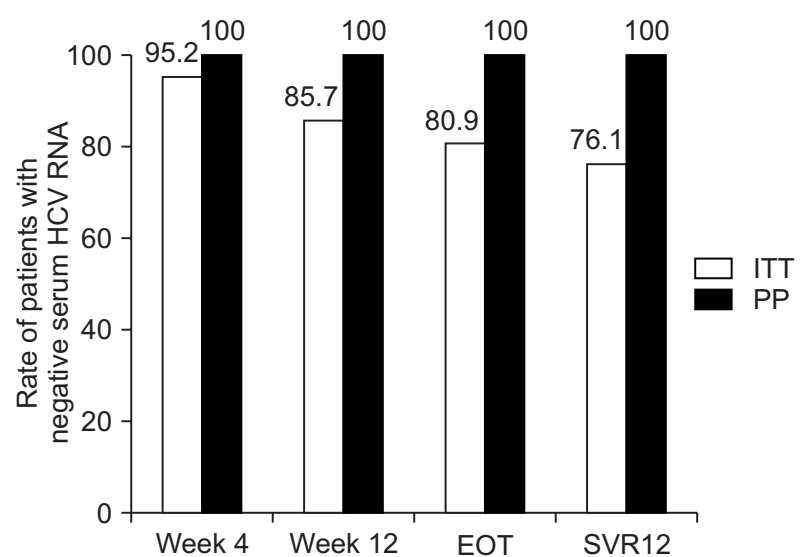

Fig. 2. SVR 12 according to intention-to-treat (ITT) and by perprotocol (PP) analysis. The overall SVR12 rate was 76.1\% in an ITT analysis and $100 \%$ in a PP analysis.

SVR12, sustained virologic response 12 weeks posttreatment; EOT, end of treatment.

up [n=1]) were excluded from the PP analysis; in that analysis, patients who completed the DCV and ASV treatment exhibited an SVR12 rate of 100\%. The virologic response rates at weeks 4, 12, and at the EOT were all 100\% respectively (Fig. 2).

\section{Safety and tolerability}

Seventeen patients $(80 \%, 17 / 21)$ completed 24 weeks of treatment. The majority of AEs were of mild or moderate severity; the most common ( $>5 \%$ ) were dizziness, dyspnea, and constipation. There was no episode of elevated ALT or total bilirubin during study period. However, grade 3 anemia (hemoglobin levels $\leq 8.9 \mathrm{~g} / \mathrm{dL}$ ) was occurred in four patients (19\%), possibly due to underlying renal disease (Table 3).

Three patients discontinued the study medication due to AEs (14.2\%). AEs related study discontinuation was dizziness, dyspnea, and neutropenia. All patients showed virologic response (HCV RNA $<15 \mathrm{IU} / \mathrm{mL}$ ) at week 4. Patients with dizziness or dyspnea showed no evidence of abnormality of electrocardiography, chest X-ray, and medication with risk of drug-drug interaction. They stopped study medication due to symptoms related nonadherence. One of whom discontinued the treatment died. This patient showed neutropenia (absolute neutrophil count <500). After discontinuation of study drugs, he was diagnosed, by bone marrow biopsy, with acute myeloid leukemia; unfortunately, this individual died from the disease. However, this was not associated with the study medication.

\section{DISCUSSION}

In the era of interferon treatment, treatment of HCV infection in patients on hemodialysis is hampered by poor tolerability and low SVR rates. Recently, new paradigms shift of HCV treatment from pegylated interferon-based therapy to DAAs showed high SVR rates, less toxicity, and good tolerability. DAAs have
Table 3. Safety of Treatment $(\mathrm{n}=21)$

\begin{tabular}{ll}
\hline \multicolumn{1}{c}{ Treatment safety } & No. (\%) \\
\hline Serious adverse events & $10(47.6)$ \\
Death* & $1(4.7)$ \\
Discontinued study medication due to adverse event & $3(14.2)$ \\
Discontinued study medication due to & 0 \\
drug-related adverse event & \\
Hemoglobin & \\
Grade $1(10-10.9 \mathrm{~g} / \mathrm{dL})$ & $13(62)$ \\
Grade 2 (9.0-9.9 g/dL) & $4(19)$ \\
Grade 3 (7.0-8.9 g/dL) & $4(19)$ \\
Grade 4 (<7.0 g/dL) & 0 \\
Total bilirubin > ×5 baseline & 0 \\
Elevated AST or ALT > x5 baseline & 0 \\
Adverse events & \\
Dizziness & $2(9.5)$ \\
Dyspnea & $2(9.5)$ \\
Constipation & $2(9.5)$ \\
Diarrhea & $1(4.7)$ \\
Atrial fibrillation & $1(4.7)$ \\
Neutropenia & $1(4.7)$ \\
Pneumonia & $1(4.7)$ \\
Gastric ulcer & $1(4.7)$ \\
\hline
\end{tabular}

AST, aspartate aminotransferase; ALT, alanine aminotransferase.

*This case was not associated with study medication. The patient expired due to acute leukemia.

been used successfully in difficult-to-treat HCV patients including those with treatment failure, decompensated cirrhosis, and post-transplantation. We report that DCV and ASV combination therapy is efficacious and safe in patients with genotype $1 \mathrm{~b}$ HCV infection on hemodialysis.

In this study, 21 patients with HCV genotype $1 \mathrm{~b}$ infection on hemodialysis received 24 weeks of DCV and ASV. The SVR12 rate was $76.1 \%$ with follow-up loss after EOT $(n=1)$, discontinuation due to AEs unrelated to study medications $(n=3)$, and virologic breakthrough $(n=1)$. Indeterminate NS5A polymorphisms at baseline might have played a role in the virologic breakthrough. However, all patients who followed up at 12 weeks after EOT achieved 100\% SVR12. Therefore, DCV and ASV combination therapy may be effective in the treatment of genotype $1 \mathrm{~b}$ infected HCV patients on hemodialysis

The SVR12 rates in this study are not consistent with those reported in genotype $1 \mathrm{~b}$ HCV patients with normal renal function. ${ }^{12,21}$ In real-life clinical practice of Korea, the SVR12 rate is $89.5 \%$ in all patients and $91.5 \%$ in those without baseline NS5A resistance-associated variants (NS5A RAVs) mutations. ${ }^{12}$ In Japan, the SVR12 rate of patients with HCV genotype $1 \mathrm{~b}$ and ESRD is reportedly $95.5 \%$ to $100 \% .^{22,23}$ This inconsistency of our study can explain as following: First, it is important to clarify 
and identify the baseline NS5A polymorphism before treating DAAs. Second, it is also an issue to monitor and manage the tolerability and safety in difficult-to-treat patients.

Some naturally occurring HCV polymorphisms that reduce DAA activity in vitro may affect the SVR12 rate, depending on the antiviral drugs used. ${ }^{24}$ The prevalence of NS5A RAVs in the HCV genotype $1 \mathrm{~b}$-infected Korean patients is $10 \%$ to $13 \%$. $^{12,21}$ The presence of baseline NS5A polymorphisms, including L31F/ $\mathrm{I} / \mathrm{M} / \mathrm{V}$ and $\mathrm{Y} 93 \mathrm{H}$, reduced the SVR12 rate in HCV genotype 1binfected patients treated with DCV and ASV to 35\%-40\%, compared to $95 \%$ in those without such polymorphisms. ${ }^{21}$ The sole patient who showed treatment failure in our study, this patient was a 62-year-old man without cirrhosis, and indeterminant NS5A mutation status at baseline despite repeated testing. He showed a partial virological response at week 4 (HCV RNA 5,350 IU/mL compared to $143,000 \mathrm{IU} / \mathrm{mL}$ at baseline). However, his serum HCV RNA level at week 12 showed a virological breakthrough (a confirmed increase in the HCV RNA more than $1 \log 10(42,300 \mathrm{IU} / \mathrm{mL})$ from the nadir), although the combination therapy has continued. Therefore, NS5A RAVs should be identified at baseline, and other DAA regimens may be more suitable for patients with indeterminate RAVs.

Tolerability is also an important issue in difficult-to-treat patients. The patients who discontinued the study medication experienced AEs including dizziness, dyspnea, and neutropenia. These AEs were likely not associated with the study medications. However, these patients have relatively more comorbidities (e.g., hypertension and DM) and a high risk of drug-drug interactions between DAAs and medications prescribed for underlying diseases, even if pharmaceutical reactions are considered. Thus, close monitoring of tolerability and safety is need when treating $\mathrm{HCV}$ patients on hemodialysis.

Our study had several strengths and limitations. First, this study is the first prospective Korean study of HCV-infected patients on hemodialysis. There are few data to guide dosing and regimens of DAAs in ESRD $(\mathrm{Cr}<30 \mathrm{~mL} / \mathrm{min}) .{ }^{6,25}$ This combination regimen may be effective and safe in HCV-infected patients on hemodialysis. However, the number of patients was small, and so a further study is needed. Second, our study highlights that it is important to identify the candidates who can benefit from DCV and ASV combination therapy based on baseline RAVs testing of HCV genotype $1 \mathrm{~b}$, to maximize SVR rates and to monitor the tolerability during treatment throughout the difference of SVR12 rate between ITT and PP analysis (76.1\% vs $100 \%$, respectively). Third, the limitation of this trial is that DCV-ASV combination in genotype $1 \mathrm{~b}$ HCV infection is no more recommended regimen, in terms of longer duration of treatment periods, pre-existing and development of resistance associate variants (RAV) of NS5A resulting low achievement of SVR12 compared with recently approved DAA regimens in Korea. Elbasvir+grazoprevir, ombitasvir/paritaprevir/ ritonavir+dasabuvir and glecaprevir+pibrentasvir combinations are better available regimens for Korean patients with genotype 1b, chronic hepatitis C and ESRD. ${ }^{6,25,26}$

In conclusion, our data demonstrate the efficacy of the DCVASV combination in the treatment of genotype $1 \mathrm{~b}$ infected HCV patients on hemodialysis. To maximize SVR rates, it is important to identify patients who will benefit from DCV and ASV combination therapy by baseline RAVs testing of HCV genotype 1b. Finally, close monitoring of safety and tolerability is necessary during DCV-ASV combination treatment of HCV-infected patients on hemodialysis.

\section{CONFLICTS OF INTEREST}

No potential conflict of interest relevant to this article was reported.

\section{ACKNOWLEDGEMENTS}

This study was funded by a grant from Bristol Myers Squibb. The study sponsor had no oversight or influence study design, in the collection, analysis, and interpretation of data or drafting the manuscript.

Author contributions: All authors recruited patients and collected data or were involved in data interpretation. All authors were involved in drafting the manuscript and approved the final version of the manuscript.

\section{REFERENCES}

1. Westbrook RH, Dusheiko G. Natural history of hepatitis C. J Hepatol 2014;61(1 Suppl):S58-S68.

2. Mohd Hanafiah K, Groeger J, Flaxman AD, Wiersma ST. Global epidemiology of hepatitis $\mathrm{C}$ virus infection: new estimates of age-specific antibody to HCV seroprevalence. Hepatology 2013;57:1333-1342.

3. Johnson DW, Dent H, Yao Q, et al. Frequencies of hepatitis B and $\mathrm{C}$ infections among haemodialysis and peritoneal dialysis patients in Asia-Pacific countries: analysis of registry data. Nephrol Dial Transplant 2009;24:1598-1603.

4. Jin DC, Yun SR, Lee SW, Han SW, Kim W, Park J. Current characteristics of dialysis therapy in Korea: 2015 registry data focusing on elderly patients. Kidney Res Clin Pract 2016;35:204-211.

5. Jadoul M, Poignet JL, Geddes C, et al. The changing epidemiology of hepatitis C virus (HCV) infection in haemodialysis: European multicentre study. Nephrol Dial Transplant 2004;19:904-909.

6. Pockros PJ, Reddy KR, Mantry PS, et al. Efficacy of direct-acting antiviral combination for patients with hepatitis $\mathrm{C}$ virus genotype 1 infection and severe renal impairment or end-stage renal disease. Gastroenterology 2016;150:1590-1598.

7. Park H, Adeyemi A, Henry L, Stepanova M, Younossi Z. A metaanalytic assessment of the risk of chronic kidney disease in patients with chronic hepatitis C virus infection. J Viral Hepat 
2015;22:897-905.

8. Lai TS, Lee MH, Yang HI, et al. Hepatitis C viral load, genotype, and increased risk of developing end-stage renal disease: REVEALHCV study. Hepatology 2017;66:784-793.

9. Fabrizi F, Takkouche B, Lunghi G, Dixit V, Messa P, Martin P. The impact of hepatitis $C$ virus infection on survival in dialysis patients: meta-analysis of observational studies. J Viral Hepat 2007;14:697-703.

10. Carvalho-Filho RJ, Feldner AC, Silva AE, Ferraz ML. Management of hepatitis $\mathrm{C}$ in patients with chronic kidney disease. World $\mathrm{J}$ Gastroenterol 2015;21:408-422.

11. Morales JM, Bloom R, Roth D. Kidney transplantation in the patient with hepatitis $\mathrm{C}$ virus infection. Contrib Nephrol 2012;176:77-86.

12. Cho BW, Kim SB, Song IH, et al. Efficacy and safety of daclatasvir plus asunaprevir for Korean patients with HCV genotype Ib infection: a retrospective multi-institutional study. Clin Mol Hepatol 2017;23:51-56.

13. Garimella T, Wang R, Luo WL, et al. Single-dose pharmacokinetics and safety of daclatasvir in subjects with renal function impairment. Antivir Ther 2015;20:535-543.

14. Eley T, Garimella T, Li W, Bertz RJ. Asunaprevir: a review of preclinical and clinical pharmacokinetics and drug-drug interactions. Clin Pharmacokinet 2015;54:1205-1222.

15. AASLD/IDSA HCV Guidance Panel. Hepatitis C guidance: AASLDIDSA recommendations for testing, managing, and treating adults infected with hepatitis C virus. Hepatology 2015;62:932-954.

16. Korean Association for the Study of the Liver. KASL clinical practice guidelines: management of hepatitis C. Clin Mol Hepatol 2016;22:76-139.

17. European Association for the Study of the Liver. EASL recommendations on treatment of hepatitis C 2016. J Hepatol 2017;66:153194.

18. Ahn SB, Jun DW, Kim SG, et al. Efficacy and safety of pegylated interferon base treatment in patients with chronic hepatitis $\mathrm{C}$ on dialysis. Eur J Intern Med 2015;26:292-296.

19. Liu CH, Huang CF, Liu CJ, et al. Pegylated interferon-alpha2a with or without low-dose ribavirin for treatment-naive patients with hepatitis $C$ virus genotype 1 receiving hemodialysis: a randomized trial. Ann Intern Med 2013;159:729-738.

20. Kumada H, Suzuki F, Suzuki Y, et al. Randomized comparison of daclatasvir + asunaprevir versus telaprevir + peginterferon/ribavirin in Japanese hepatitis C virus patients. J Gastroenterol Hepatol 2016;31:14-22.

21. McPhee F, Suzuki Y, Toyota J, et al. High sustained virologic response to daclatasvir plus asunaprevir in elderly and cirrhotic patients with hepatitis $\mathrm{C}$ virus genotype $1 \mathrm{~b}$ without baseline NS5A polymorphisms. Adv Ther 2015;32:637-649.

22. Toyoda H, Kumada T, Tada T, et al. Safety and efficacy of dual direct-acting antiviral therapy (daclatasvir and asunaprevir) for chronic hepatitis $\mathrm{C}$ virus genotype 1 infection in patients on hemodialysis. J Gastroenterol 2016;51:741-747.

23. Suda G, Kudo M, Nagasaka A, et al. Efficacy and safety of daclatasvir and asunaprevir combination therapy in chronic hemodialysis patients with chronic hepatitis C. J Gastroenterol 2016;51:733740.

24. Kwo P, Gane EJ, Peng CY, et al. Effectiveness of elbasvir and grazoprevir combination, with or without ribavirin, for treatmentexperienced patients with chronic hepatitis C infection. Gastroenterology 2017;152:164-175.

25. Roth D, Nelson DR, Bruchfeld A, et al. Grazoprevir plus elbasvir in treatment-naive and treatment-experienced patients with hepatitis $C$ virus genotype 1 infection and stage 4-5 chronic kidney disease (the C-SURFER study): a combination phase 3 study. Lancet 2015;386:1537-1545.

26. Gane E, Lawitz E, Pugatch D, et al. Glecaprevir and pibrentasvir in patients with HCV and severe renal impairment. N Engl J Med 2017;377:1448-1455. 